\title{
Synthesis and characterization of semi-permeable graphene/graphene oxide membranes for water desalination
}

\author{
Grzegorz Romaniak $^{1,2, *}$ (), Konrad Dybowski ${ }^{1}$ (1), Agata Jeziorna ${ }^{1}$ (D), Piotr Kula ${ }^{1}$ (1), and \\ Tomasz Kaźmierczak ${ }^{2}$ (D) \\ ${ }^{1}$ Faculty of Mechanical Engineering, Institute of Materials Science and Engineering, Lodz University of Technology, 1/15 \\ Stefanowskiego St., 90-924 Lodz, Poland \\ ${ }^{2}$ Amii Sp. z o.o., 22 Techniczna St., 92-518 Lodz, Poland
}

Received: 21 January 2020

Accepted: 3 April 2020

Published online:

15 April 2020

(C) The Author(s) 2020

\begin{abstract}
In this paper, we present novel semi-permeable graphene-based membranes. Composite filters were designed and fabricated on polysulfone porous scaffolding using combinations of polycrystalline large-area High Strength Metallurgical Graphene (HSMG®), graphene oxide, hydrazine and an in-situ interfacial polymerized polyamide. The naturally occurring defects in HSMG® (which were tenths of a nanometer) were the clue in fabricating a filtering membrane. The performance of graphene membranes was evaluated in forward osmosis test. The prepared composites were proved to be semi-permeable membranes with great ions blocking efficiency (over 95\%) and water flux only one order of magnitude lower than the commercial reverse osmosis membranes. The experiments' results demonstrated that the solutions proposed in this work indicate that graphene-based membranes can be used in water treatment technology.
\end{abstract}

\section{Introduction}

Life of most living organisms on Earth is dependent on access to clean water. Over one billion people live in areas which suffer from water shortages [1]. For this reason, in order to ensure the right quality of potable water, various technical solutions as well as physicochemical phenomena are used to reduce the concentration of various types of impurities. The removal of ions and particles can be carried out by filtration, using reverse osmosis process, by ion exchange resins or electrodeionization, for example. Membrane processes deserve special attention as they are widely used in water treatment [2]. Membrane technologies-due to their low cost, easy maintenance and high rejection rate-have gained popularity during the last few decades and are widely used in very specific applications [3, 4].

Progress in discovering new materials and developing new nanotechnologies meant that intense

Address correspondence to E-mail: grzegorz.romaniak@edu.p.lodz.pl 
research carried out is aimed at replacing composites which are currently used and are based on polymers in membrane technology by new-generation nanomaterials such as graphene, carbon nanotubes (CNTs), graphene oxide (GO) and other 2D materials [2, 5-8]. Carbon-based nanomaterials are particularly interesting because of their unique properties, such as large surface area, large porosity range and exceptional mechanical strength. Smooth friction-free graphite walls of carbon nanotubes and graphene, as well as a fast sorption-desorption mechanism, can facilitate the transport of water molecules, ensuring high efficiency of flux separation. Unfortunately, the high-cost sourcing and difficulties in fabricating of 3D well-aligned carbon nanotube arrays-based membranes make their wide commercial use very difficult.

An important issue in membrane processes is gaining a high level of permeability per unit of pressure while providing a high rejection rate as well as resistance to oxidation and fouling [2].

The solutions proposed currently, based on 2D materials, allow working near a thermodynamic limit with a pressure which is only $10-20 \%$ higher than osmotic pressure [5].

Desalination using nanoporous graphene membranes and composites with graphene oxide is realized by the exclusion of ions by filtration, which is connected with the size of pores, the distance between the graphene sheets or electrostatic interactions. The distance should fall within a range of $0.3-0.7 \mathrm{~nm}$ to make water flow possible (the water molecule diameter of $0.27 \mathrm{~nm}$ [8]) while separating pollution ions. Defect-free graphene sheet, according to information from the literature, is an ideal barrier for gases and liquids [6]. Moreover, this material has exceptional chemical [9] as well as mechanical properties [10] and is the thinnest possible layer with a thickness of only 1 atom. That is why defects purposely made in graphene with the use of plasma, laser beam perforation and selective oxidation [11], electron beam [12], ionic etching [13, 14] and ion bombardment [15] give the possibility to make a layer which is nanoporous and can be used as a membrane.

Polycrystalline graphene synthesized on the liquid copper (HSMG ${ }^{\circledR}$ - High Strength Metallurgical Graphene) has special properties as defects with a size of a tenth of a nanometer can be found in its structure $[16,17]$. Aside from the desired size defects, the largearea HSMG ${ }^{\circledR}$ graphene sheets also contain large defects resulting from synthesis and/or transfer onto another substrate, which make effective separation impossible.

Among the methods of sealing these defects, it is worth mentioning the interfacial polymerization of nylon from two precursors separated by the membrane [7], the deposition of metals and the metal oxide which uses atomic layer deposition (ALD) in discontinuities on defects borders [8].

In several studies on composite graphene membranes, we also met membranes based on graphene oxide or graphene powder, manufactured from liquid suspension and strengthened with binder [18] as well as layers of graphene, graphene oxide or their combination applied on polymeric nanofiber surface [19]. The method of laying graphene oxide on the surface has a huge impact on the positioning and orientation of material flaxes. Gravitational coating of load-bearing oxide causes chaotic distribution in the layer created and large uncontrolled porosity [20]. A high GO orientation degree is gained using a pressure-assisted method or spin coating [21]. Due to the water pressure applied, membranes have to be manufactured on scaffoldings, e.g., metallic, ceramic or polymeric ones, depending on the application in forward osmosis or reverse osmosis [22].

In our study, graphene oxide was used as an effective sealing matter, which can form a thin and strong layer, especially when its flakes were subjected to nanostructurization.

In the presented work, centimeter-scale membranes with a large-area polycrystalline HSMG ${ }^{\circledR}$ graphene transferred onto polysulfone (PSU) commercial microfiltration membranes were prepared. We utilized novel composite membranes' advantages which result from graphene synthesis on liquid copper. Detected subnanometric defects are a clue for the use of HSMG ${ }^{\circledR}$ graphene in filtration technology. However, due to the large defects in graphene generated during transfer on porous substrates, such membranes are not effective. These defects must be eliminated. For this purpose, graphene oxide, hydrazine and/or nylon were applied. Graphene oxide in various types of configuration-with additional nanostructurization with hydrazine and/or nylon interfacial polymerization sealing-was prepared to obtain the best water permeance and ion rejection rate. Membranes under the influence of an osmotic pressure gradient caused by a $\mathrm{NaCl}$ concentration difference revealed their semi-permeable 
properties, such as ion transport blocking while allowing water flux to go through the membrane. This shows that prepared composites have the potential to be used in water treatment technology.

\section{Experimental}

\section{Characterization of materials}

\section{Graphene HSMG®}

Graphene for building the composite was obtained using the metallurgical method. Graphene HSMG® synthesis and substrate preparation were carried out according to the procedure which was made a patent (Method of producing graphene from liquid metal, US 9284 640, USA) [16, 17, 23]. The substrate for crystallization of graphene was a $\mathrm{Cu}-\mathrm{Ni}$ sheet. A nickel foil with a thickness of $0.15 \mathrm{~mm}$ was coated with electroplated copper in a saturated solution of copper sulfate pentahydrate $\left(\mathrm{CuSO}_{4} \cdot 5 \mathrm{H}_{2} \mathrm{O}\right)$. The current density was $2 \mathrm{~A} \mathrm{dm}^{-2}$, the processing time lasted $6 \mathrm{hrs}$, and the obtained thickness equalled $0.2 \mathrm{~mm}$. Monolayer sheets of HSMG® graphene were produced in a vacuum furnace.

The following process parameters were applied:

- heating in a vacuum (pressure $10 \mathrm{~Pa}$ ) to a temperature of $1060{ }^{\circ} \mathrm{C}$,

- carburizing of the $\mathrm{Cu}-\mathrm{Ni}$ substrate in an ethyleneacetylene-hydrogen gas mixture (four alternating stages of gas dosing and isothermal annealing, during which the carburizing atmosphere was dosed during $5 \mathrm{~s}$, followed by annealing in a vacuum for $600 \mathrm{~s}$ ) at a temperature of $1060{ }^{\circ} \mathrm{C}$,

- heating in an argon and hydrogen gas mixture to a temperature of $1100{ }^{\circ} \mathrm{C}$ under $2 \mathrm{kPa}$ pressure

- isothermal annealing at a temperature of $1100{ }^{\circ} \mathrm{C}$ for $5 \mathrm{~min}$,

- cooling in an argon and hydrogen atmosphere to an ambient temperature.

The graphene produced was subjected to qualitative tests by optical microscopy on a $\mathrm{Cu}-\mathrm{Ni}$ substrate. The surface was washed with water before analysis. The exposure time was about $2 \mathrm{~min}$. Copper may be oxidized this way in places not covered with graphene, thus revealing macroscopic discontinuities in the graphene layer. The purpose of this operation was to select substrates with graphene for further proceedings in the planned tests, i.e., without signs of any change in copper color. Randomly selected places, after treatment with $0.1 \mathrm{M} \mathrm{FeCl}_{3}$ solution (exposure time was $5 \mathrm{~s}$ ), were analyzed using a microscope (Fig. 1).

$\mathrm{FeCl}_{3}$, as a strong oxidant, reacts very quickly with copper, hence why it is used to remove copper substrates after graphene synthesis. $\mathrm{FeCl}_{3}$ gets under the graphene layer, where it penetrates and digests the metal. Dark spots which appear in the microscopic image (Fig. 1) reflect discontinuities in the layerdefinitely extended by the residual metal etching products.

Next, Raman spectroscopy was used for the investigation. The measurements were made using a laser wavelength of $532 \mathrm{~nm}$; the laser power

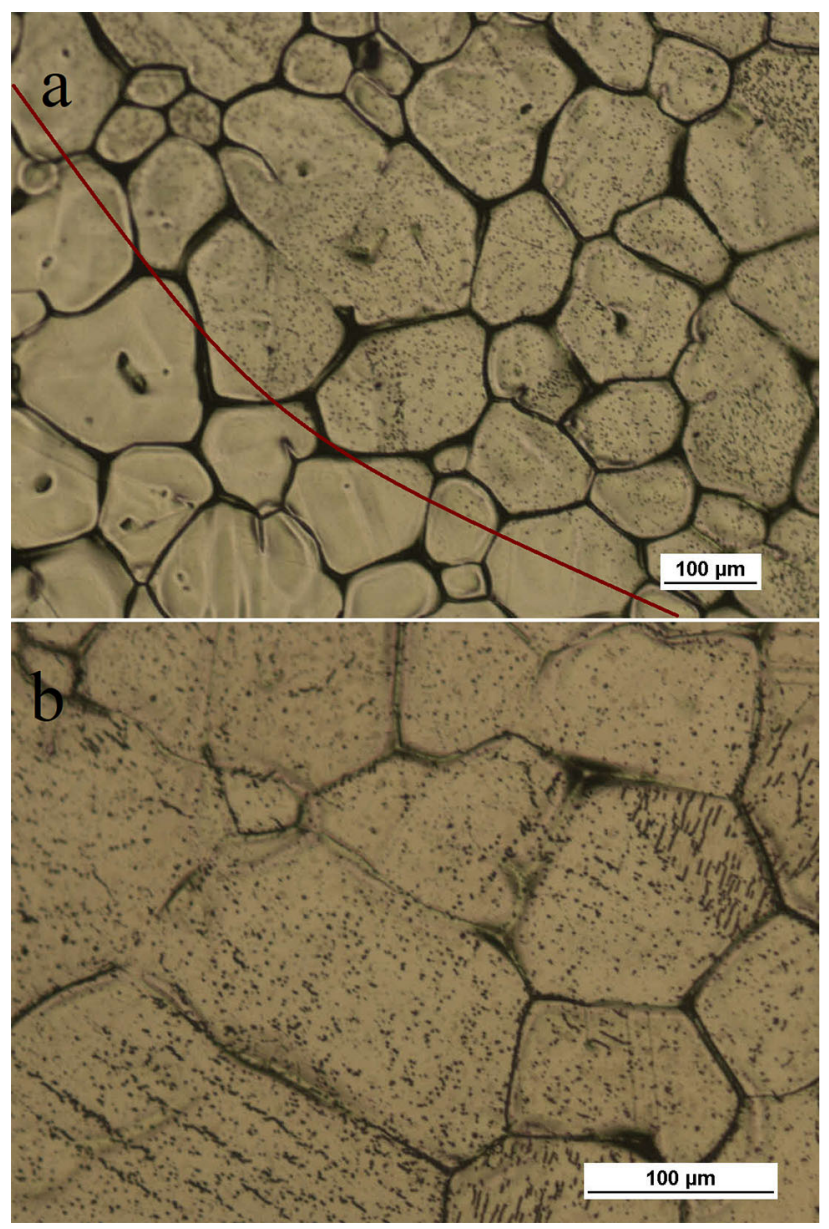

Figure $1 \mathrm{Cu}-\mathrm{Ni}$ substrate surface with a graphene layer-results of the treatment with $0.1 \mathrm{M} \mathrm{FeCl}_{3}$ solution. Optical microscope image at different magnifications. a Visible boundary of the $\mathrm{FeCl}_{3}$ operating field and intact area of the copper matrix (a border marked with the red line); $\mathbf{b}$ the place after act of $\mathrm{FeCl}_{3}$ under higher magnification. 
delivered to the sample was $10 \mathrm{~mW}$. When analyzing the Raman spectra of the graphene, attention was paid to the presence and the intensity of graphenespecific peaks (G $1580 \mathrm{~cm}^{-1}$ and 2D $2690 \mathrm{~cm}^{-1}$ ) as well as to the peaks indicative of the scale of defects within the graphene structure (D $1350 \mathrm{~cm}^{-1}$ and $\mathrm{D}^{\prime}$ $1620 \mathrm{~cm}^{-1}$ ). The Raman spectra obtained showed the presence of a graphene monolayer on the growth substrate (Fig. 2). A TEM examination was used to assess the nanostructure of the graphene layers produced. A TEM Talos F200X from FEI was used in the research. This is an atomic resolution microscope with a maximum voltage which accelerates electrons of $200 \mathrm{kV}$. Based on these studies, nanostructural defects of a size from 0.2 to $0.5 \mathrm{~nm}$ were identified (Fig. 3).

\section{Polysulfone membrane}

MicroPES ${ }^{\circledR}$ 1F EL porous support substrate-made by Membrane $\mathrm{GmbH}$, Germany-was used to prepare composite graphene membranes. Membrane thickness equals $110 \mu \mathrm{m}$ with an average pore size of $0.2 \mu \mathrm{m}$ and water permeability at a temperature of $25{ }^{\circ} \mathrm{C}$ of $\geq 10 \mathrm{ml} \mathrm{min}{ }^{-1} \mathrm{~cm}^{-2} \mathrm{bar}^{-1}$. The membranes were characterized by varying porosity in the cross section.

Figure 4a shows one side of this membrane-the major porosity side. The pore diameter on this side of the membrane is from 0.2 to $1.5 \mu \mathrm{m}$. The density of the pores is $0.26 \times 10^{6} \mathrm{~mm}^{-2}$. Figure $4 \mathrm{~b}$ shows the side of a polysulfone membrane with minor porosity. The pore diameter on this side of the membrane is from 0.1 to $1.0 \mu \mathrm{m}$. The density of the pores is $1.05 \times 10^{6} \mathrm{~mm}^{-2}$. We always transferred graphene to the surface with minor porosity. Exactly the same type of polysulfone membranes was used in each test.

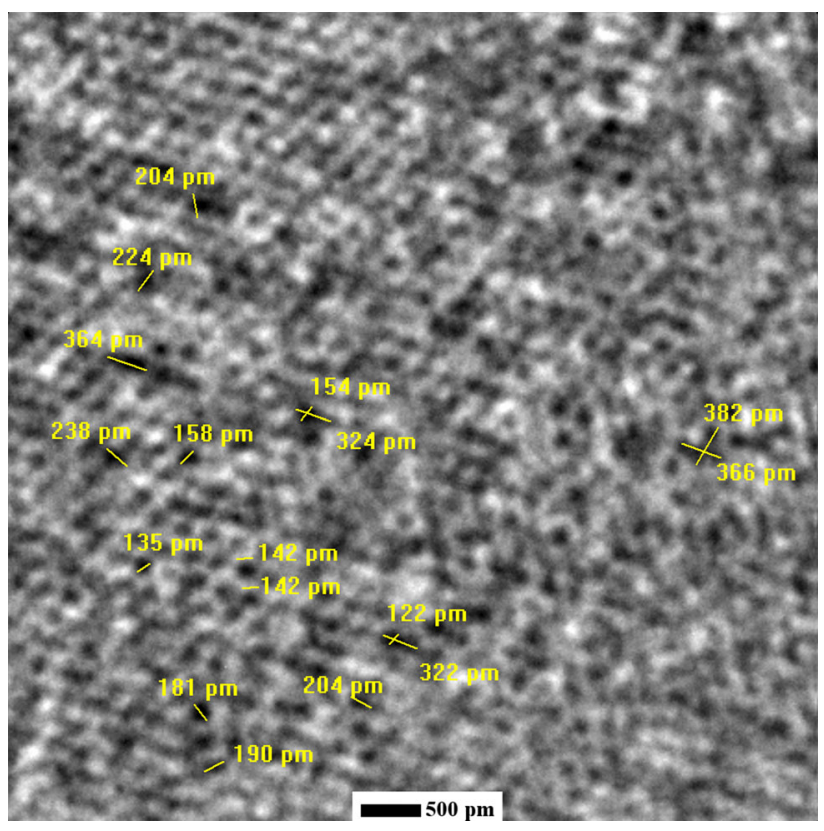

Figure 3 Point defects of the graphene HSMG ${ }^{\circledR}$ atomic structure. HRTEM image.

\section{GO and $r G O$ platelets}

Graphene oxide with $0.1 \%$ dispersion in water (1 mg/1 ml) was purchased from the Advanced Graphene Products Company, Poland. FTIR analysis confirmed the purity and oxidation state of the material used in our studies and provided an excellent reference to the result obtained by GO flakes hydrazine treatment on the polysulfone membrane (Fig. 5c).

$20 \mu \mathrm{l}$ of a $1 \%$ aqueous hydrazine solution was used per $1 \mathrm{~cm}^{2}$ of the membrane surface. The hydrazine solution was pipetted gently on top of the composite membrane and left to react with graphene oxide for at least $120 \mathrm{~min}$. The membrane was then rinsed several times by immersion in a water bath and was then air-dried.

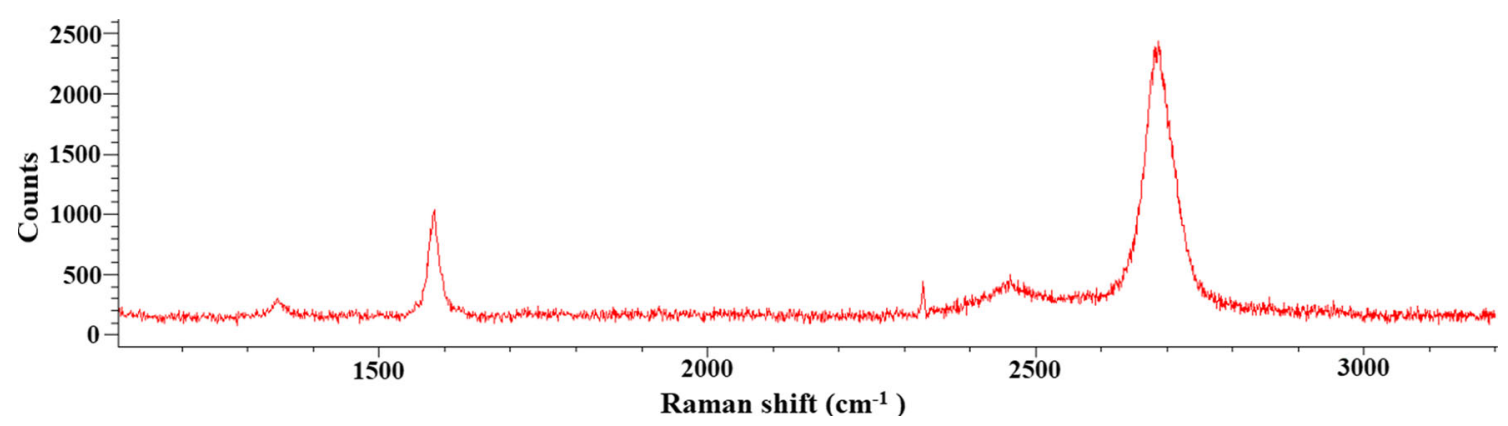

Figure 2 Raman spectrum of graphene. 


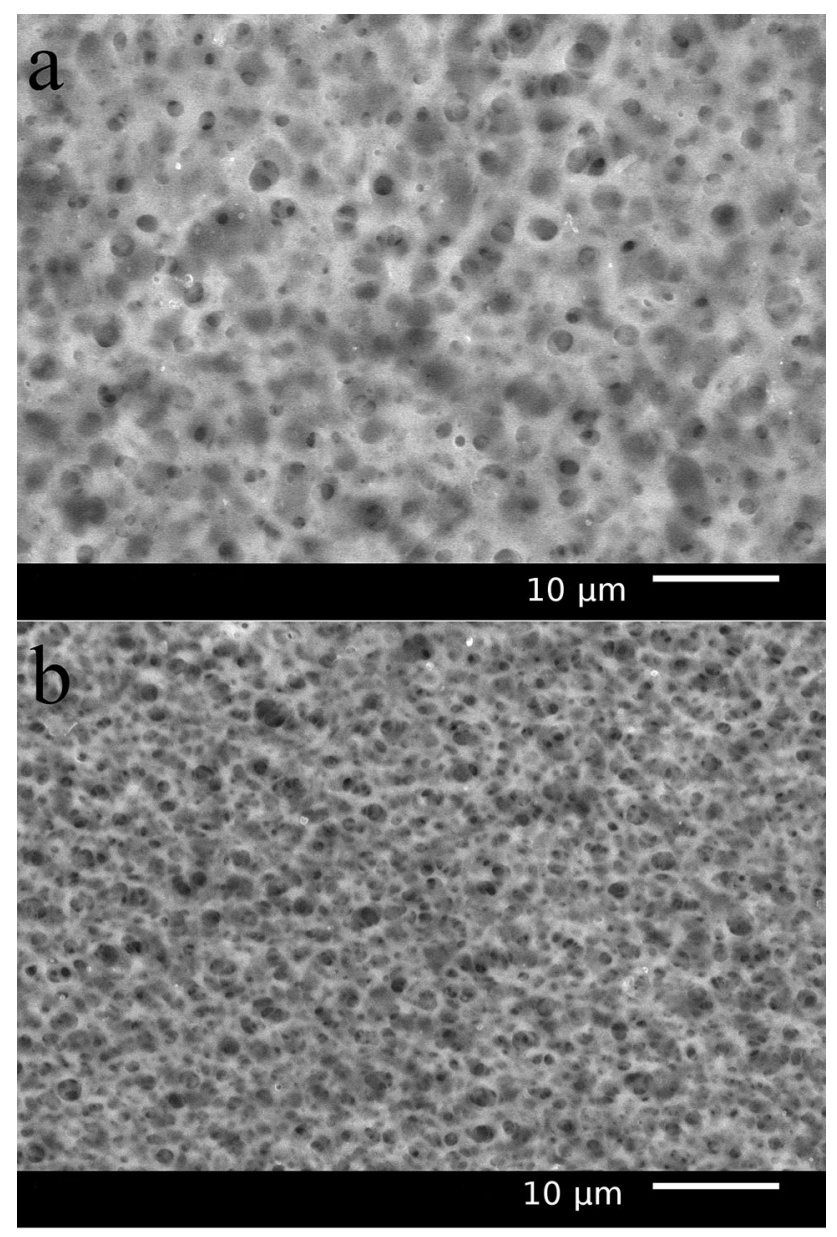

Figure 4 The polysulfone membrane structure. a The major porosity side, $\mathbf{b}$ the minor porosity side. SEM SE image, LSEI mode, $20 \mathrm{kV}$.

The $1 \%$ hydrazine solution was prepared by diluting commercial $80 \%$ hydrazine hydrate from POCh, Poland, immediately before use.

\section{Solvents and reagents}

Monomers used in the synthesis of polyamide in graphene defects on the surface of composite membranes were 98\% 1,6-diaminohexane (Alfa Aesar, Thermo Fisher Scientific, Great Britain) and 92\% sebacoyl chloride (Sigma-Aldrich). Cyclohexane (Chempur, Poland) was a solvent of sebacoyl chloride.

Poly(methyl methacrylate) (PMMA) with a molecular weight of $996000 \mathrm{~g} / \mathrm{mol}$ of the Aldrich Chemistry Company, dissolved in chlorobenzene (Chempur, Poland) with a concentration of $0.05 \mathrm{~mol} \mathrm{dm}^{-3}(4.6 \mathrm{~g}$ of PMMA-100 $\mathrm{ml}$ of chlorobenzene), was applied to coating the graphene substrate in the transfer procedure.

The solvent used to dissolve and remove the PMMA foil (graphene support during the transfer on the polysulfone membrane) was 2-propanol with a technical grade of $70 \%$ (Chempur, Poland).

Sodium chloride was used in the osmotic transport experiments (Chempur, Poland). The starting concentration of $\mathrm{NaCl}$ in the water was $\mathrm{c}=0.2 \mathrm{~mol} \mathrm{dm}^{-3}$.

\section{Construction and characterization of semi- permeable composite membranes}

The HSMG® graphene layer is the basic and active element of composite filtration membranes. Separation of the HSMG ${ }^{\circledR}$ graphene from the growth matrix, its transfer to the target substrate-depending on the role of this matter-and the purpose of using the constructed composite are necessary to take advantage of the extraordinary properties of graphene. In the case of designed filters, the polysulfone membrane is a carrier substrate for the HSMG ${ }^{\circledR}$ graphene. The most convenient method of the graphene transfer on the scaffolding is therefore using the PMMA transfer method.

Having formed a thin PMMA film directly on the growth matrix of graphene, the graphene layer was separated from the metallic substrate by electrochemical delamination [24]. High efficiency of that process is its main advantage. There is no risk of adsorbing of undesirable chemical etching products on the graphene surface and in the graphene defects [25]. Moreover, this method allows to use repeatedly the $\mathrm{Cu} / \mathrm{Ni}$ matrix in the growth of HSMG ${ }^{\circledR}$ graphene as well as in the delamination cycles. In the case of graphene transfer to the polysulfone membrane, the polymeric support was removed in isopropanol vapors because the polysulfone could be damaged by acetone, which is typically used to dissolve PMMA.

The multiple transfer was applied by overlapping three layers of graphene in order to eliminate defects in the graphene layer arising during the transfer as well as those resulting from the specifics of the synthesis of the graphene on the liquid substrate. Another solution was to cover any defects with graphene oxide flakes with the option of cross-linking with hydrazine [26]. The next concept was using polyamide synthesis in discontinuities of the composite filtration membrane built on the basis of graphene and graphene oxide. 
Figure 5 SEM, AEE mode images of: a-a graphene (GR) on PSU, chosen purposely with large defects to show better the mechanism of sealing; $\mathbf{b}$ - a graphene on PSU with graphene oxide (GO) coating; c - a graphene on PSU with GO coating, nanostructurized with hydrazine; Scale bar $200 \mu \mathrm{m}$.
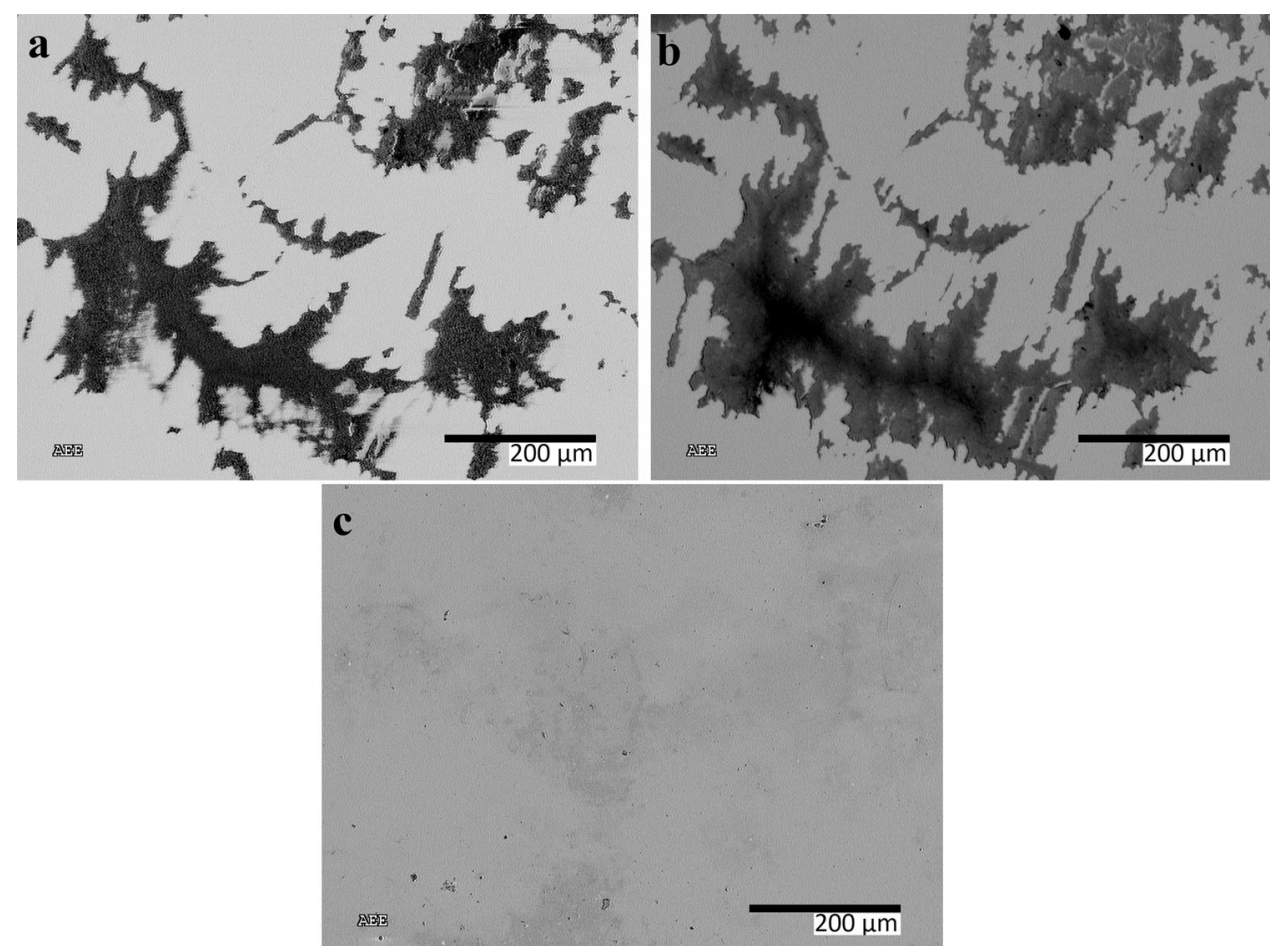

\section{Graphene transfer on Polysulfone}

Graphene samples with a size of $30 \times 30\left[\mathrm{~mm}^{2}\right]$ were prepared. Coating with PMMA in chlorobenzene as a temporary carrier layer was performed by means of drop/blade coating. The polymer dried for $30 \mathrm{~min}$ at a temperature of c.a. $40{ }^{\circ} \mathrm{C}$ formed a thin flexible film. Delamination of the graphene on the polymeric support was carried out using a homemade halfautomatic laboratory station in $0.5 \mathrm{~mol} / 1 \mathrm{NaOH}$ solution at a constant voltage $4.5 \mathrm{~V}$ at a sample submersion angle of $35^{\circ}$ and a submersion linear speed of $0.02 \mathrm{~mm} \mathrm{~s}^{-1}$. The polymer foil with graphene was placed on wetted polysulfone membrane, pressed and annealed on a heating plate at $70{ }^{\circ} \mathrm{C}$ for $5 \mathrm{~min}$. The composite, formed in such a way, was then put into a chamber with boiling isopropanol to remove the PMMA layer supporting graphene.

\section{Sealing of defects in the graphene layer}

\section{Graphene oxide (GO) as sealing species}

The graphene layer on the polysulfone membrane was covered with graphene oxide flakes that were suspended in a water-isopropanol system $(2: 1, \mathrm{v} / \mathrm{v})$ at a concentration of $c=0.0067 \mathrm{~g} / \mathrm{ml}$ and in the amount of $20 \mu \mathrm{l}$ per $1 \mathrm{~cm}^{2}$ of the graphene surface. Each time before use, the GO suspension in the above-mentioned solvents was dispersed by means of ultrasound. The addition of isopropanol reduced the surface tension of commercially available aqueous GO suspensions, which facilitated the even distribution of GO on the hydrophobic graphene layer. Due to their hydrophilic nature, the graphene oxide flakes tended to accumulate in uncovered places of the polysulfone during evaporation of the liquids from the membrane surface.

The SEM analysis of this surface (Fig. 6) revealed that graphene oxide effectively aggregated in discontinuities of the HSMG ${ }^{\circledR}$ layer to form a more or less dense structure.

A thin coating of GO flakes was clearly visible on the surface of the polysulfone membrane uncovered with the layer of HSMG ${ }^{\circledR}$ graphene (lighter area in Fig. 6). On the continuous graphene layer, only fragments of aggregated GO flakes could be found.

The method of applying GO flakes to cover the membrane was modified to increase the efficiency of filling defects in the graphene layer. The membrane covered with the sealing mixture was exposed to a pressure of 3.5 bar for $1 \mathrm{~min}$. 


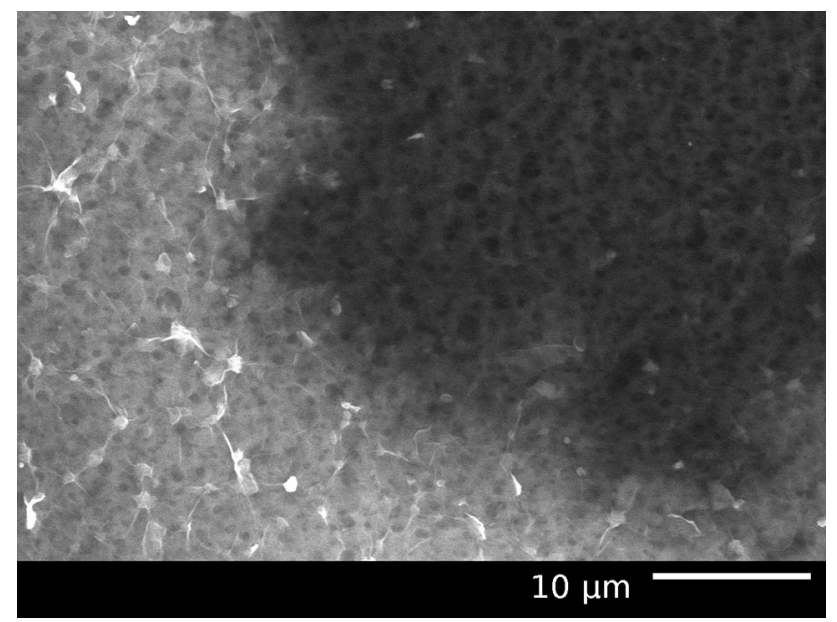

Figure 6 Polysulfone membrane coated with HSMG ${ }^{\circledR}$ graphene and filled with GO flakes. SEM image, LSEI mode, $10 \mathrm{kV}$.

\section{Sealing composition: graphene oxide with hydrazine}

The structures of the GO aggregates were strengthened by cross-linking them with hydrazine to minimize the risk of the sealing degrading during water flow. At first, a layer of GO flakes accumulated in the discontinuities of the graphene layer was treated with a $1 \%$ aqueous solution of hydrazine. In the second case, these defects were covered with a precross-linked GO flakes suspension, i.e., a previously prepared mixture of $\mathrm{GO}$ and $\mathrm{N}_{2} \mathrm{H}_{4}$. A change in the color, from brown to black, was observed in the graphene oxide. This effect resulted from reducing the number of oxygen groups in the GO flakes and reconstructing the aromatic system in the structure of the bonds between carbon atoms (change of hybridization from sp3 to sp2 and the possibility of electronic transitions). This process led to a change in the electrical properties of the sealing layer from a non-conductive (GO) to a conductive (rGO—reduced graphene oxide) one.

The continuity of the graphene layers on the nonconductive support substrate can be examined by means of the signal of electrons absorbed by the sample (AEE mode SEM). The contrast generated during the measurement is due to the differences in currents flowing through the sample depending on whether the electron beam fell within a conductive or a non-conductive area. Dark areas against a light (i.e., conductive) background indicate the structural discontinuities within the graphene layer on the polysulfone substrate (Fig. 5a, b).
Sealing composition: graphene oxide (or graphene oxide with hydrazine) and nylon

Nylon was synthesized directly on the polysulfone membrane previously covered with the HSMG ${ }^{\circledR}$ graphene layer after sealing with GO flakes with/ without hydrazine. The nylon can be prepared using interfacial polymerization. The polyamide was synthesized according to the procedure described in the literature [8]. The polysulfone membrane was placed on an aqueous amine solution (with a concentration of $c=0.02 \mathrm{~g} / \mathrm{mol}$ ) in such a way that the side covered with graphene species was in direct contact with the liquid. The other side of the membrane was gently coated with sebacoyl chloride in cyclohexane $(c=0.01 \mathrm{~g} / \mathrm{mol})$. The reaction was completed in $10 \mathrm{~min}$. Afterward, the side in contact with amine was washed with water and the side covered by sebacoyl chloride was washed with cyclohexane. The composite obtained in such a way was air-dried at room temperature.

\section{Filtration test and performance of composite membranes}

\section{Construction of an osmotic stand}

A homemade side-by-side diffusion cell (Fig. 7) for ion separation effectiveness and water flow thought membrane examination is made of two equal chambers: one for deionized water and the other for $\mathrm{NaCl}$ water solution. A membrane (1) is placed in a grip (2) with a round orifice to which containers are attached (3). Each container has an inlet (4) and a graduated glass tube (5) to read the fluid volume change.

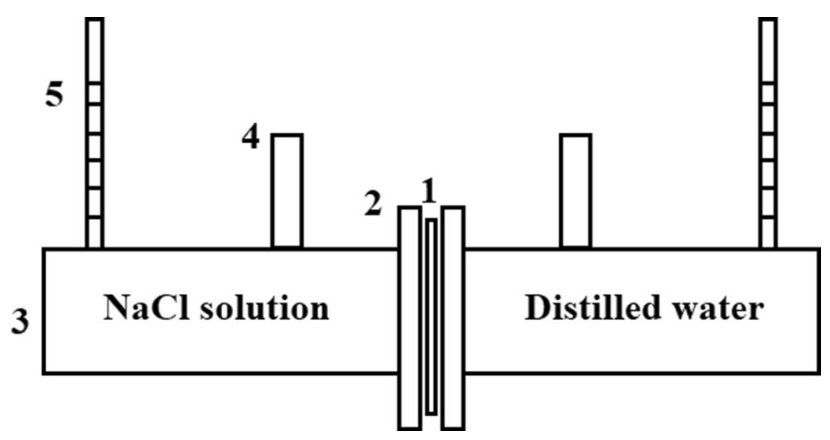

Figure 7 Scheme of a homemade side-by-side diffusion cell. 


\section{Description of the experiment}

The membrane was placed in the grip and sealed up with a semiliquid polydimethylsiloxane polymer (polydimethylsiloxane (PDMS), SYLGARD ${ }^{\mathrm{TM}} 184$ Silicone Elastomer, The Dow Corning Chemical Company, Germany). After polymerization, the PDMS took on a solid form and stabilized the membrane. The effective membrane surface area was $4 \mathrm{~cm}^{2}$. The membrane side with the graphene faced the chamber with $\mathrm{NaCl}$ water solution. The tests were conducted at a temperature of $20{ }^{\circ} \mathrm{C}$. The osmotic pressure was 9.7 bar and was calculated on the basis of the van't Hoff equation $(\pi=v c R T)$, where $v$ is the number of ions in dissociated salt, $c$ is the molar concentration of the solute, $R$ is the gas constant and $T$ is the temperature in Kelvin. Diffusion transport was studied for $24 \mathrm{~h}$. The starting concentration salt in one chamber was $0.2 \mathrm{~mol} \mathrm{dm}^{-3}$, and the opposite container was filled with degassed UV lamp-treated distilled water. The measurement of water conductivity and the change in water volume on both sides of the membrane was made after each test.

The water flux through the membrane $\mathrm{J}\left(\mathrm{ml} \mathrm{m}^{-2}\right.$ $\mathrm{h}^{-1} \mathrm{bar}^{-1}$ ) was calculated on the basis of the equation $J=\frac{V}{A t p}$, where $V$ is the change in water volume in a container $(\mathrm{ml}), A$ is the membrane active surface area $\left(\mathrm{m}^{2}\right), t$ is the time (h) and $p$ is the theoretical osmotic pressure owing to the concentration difference (bar).

\section{Results}

Different options of sealing HSMG ${ }^{\circledR}$ graphene on polysulfone membranes are listed in Table 1 . The membranes' performance was evaluated during the osmotic test. Water flux was measured for each membrane composition and for the bare PSU membrane (no change in water volume in containers) under osmotic pressure in a side-by-side osmotic cell. The ions' flux $\left(\mathrm{mol} \mathrm{m}^{-2} \mathrm{~s}^{-1}\right)$ was calculated by dividing the $\mathrm{NaCl}$ molar concentration of the solution in a container (which contained deionized water before the test) by the test time and membrane surface. Changes in the fluid level seen on the capillary of the salt chamber allowed to estimate water flow. The results of studies on the effectiveness of blocking ions, water flux and diffusion flux of $\mathrm{NaCl}$ ions through composite membranes were illustrated in the form of bar charts in Figs. 8, 9 and 10, respectively.

The osmotic tests indicated that one layer of HSMG ${ }^{\circledR}$ on the PSU membrane (the A sample) was permeable to ions (blocking efficiency of 20\%Fig. 8). The opposite results were obtained for three graphene layers on the porous substrate (the B sample) as well as for the sample with pressure-assisted GO coating (the C sample), where there was neither water nor ion flux. Two graphene layers on PSU membrane with graphene oxide between them (the D sample) resulted in a rejection rate of $60 \%$ and water flux $24 \mathrm{ml} \mathrm{h}^{-1} \mathrm{~m}^{-2} \mathrm{bar}^{-1}$. The $\mathrm{E}$ sample sealed with pre-cross-linked GO flakes blocked $69 \%$ of the ions in the osmotic test. The permeance of the membrane with graphene/GO/hydrazine (the F sample) was at a level of $15 \mathrm{ml} \mathrm{h}^{-1} \mathrm{~m}^{-2} \mathrm{bar}^{-1}$, and a diffusion flux of $5 \times 10^{-8} \mathrm{~mol} \mathrm{~m}^{-2} \mathrm{~s}^{-1}$ (Fig. 10) resulted in ion separation efficiency at a level of over $95 \%$. The membrane with defects sealed by polyamide, either with graphene oxide or graphene oxide with hydrazine treatment (the $\mathrm{G}, \mathrm{H}$ samples), resulted in an ion blocking efficiency over $90 \%$ and water permeability below $5 \mathrm{ml} \mathrm{h}^{-1} \mathrm{~m}^{-2} \mathrm{bar}^{-1}$.
Table 1 Variants of membrane compositions prepared for the osmotic test

\begin{tabular}{ll}
\hline Sample designation & Membrane composition \\
\hline A & Polysulfone + HSMG ${ }^{\circledR}$ \\
B & Polysulfone +3 HSMG $^{\circledR}$ layers \\
C & Polysulfone + HSMG $^{\circledR}+$ graphene oxide (pressure assisted) \\
D & Polysulfone + HSMG $^{\circledR}+$ graphene oxide + HSMG ${ }^{\circledR}$ \\
E & Polysulfone + HSMG $^{\circledR}+$ (graphene oxide + hydrazine solution) \\
F & Polysulfone + HSMG $^{\circledR}+$ graphene oxide + hydrazine \\
G & Polysulfone + HSMG $^{\circledR}+$ graphene oxide + nylon \\
H & Polysulfone + HSMG $^{\circledR}+$ graphene oxide + hydrazine + nylon \\
\hline
\end{tabular}


Figure 8 Ions blocking efficiency in the osmotic test.

Figure 9 Results of water flux and ion flux through composite membranes.

Figure 10 Diffusion flux of $\mathrm{NaCl}$ ions through composite membranes.

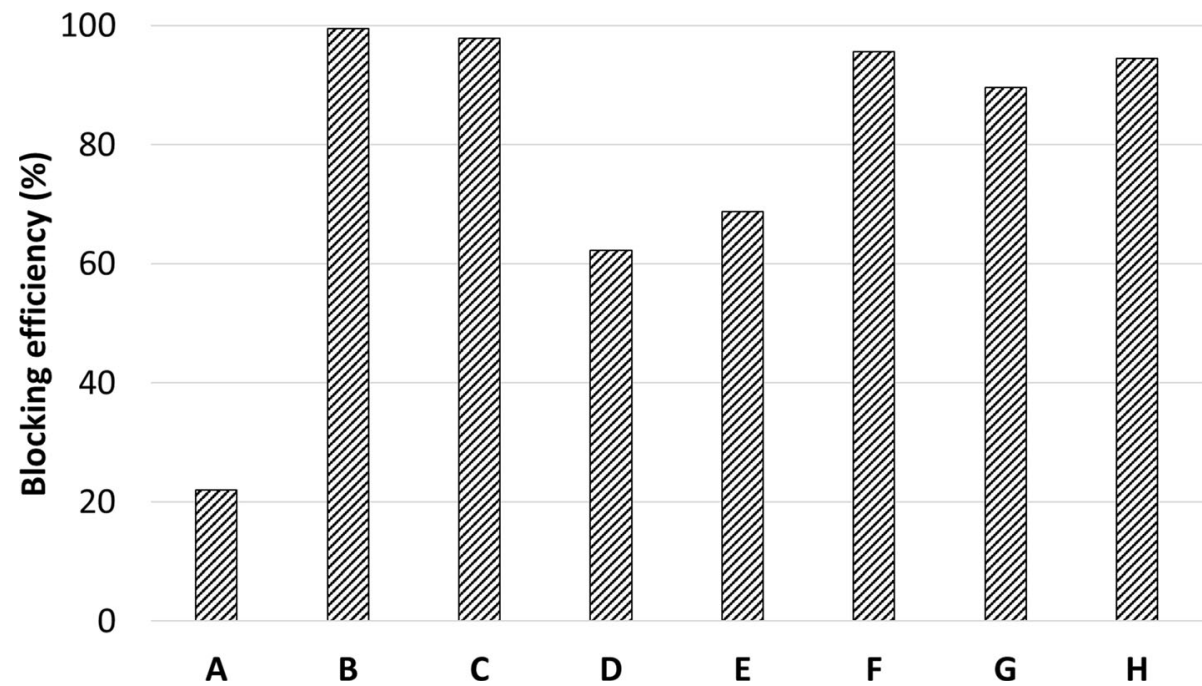

30

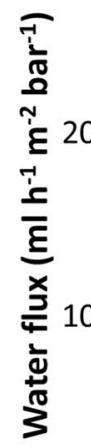

$2,5 \mathrm{E}-03$

2,0E-03

$1,5 \mathrm{E}-03$
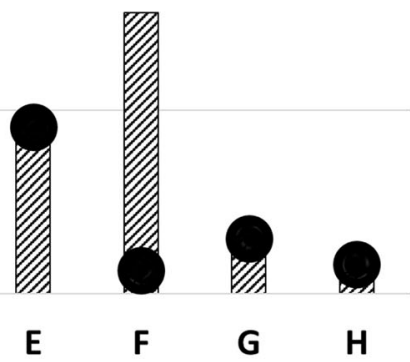

$1,0 \mathrm{E}-03$

$5,0 \mathrm{E}-04$

$0,0 E+00$

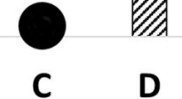

E

Water flux Ion migration flux

$1,0 \mathrm{E}-06$

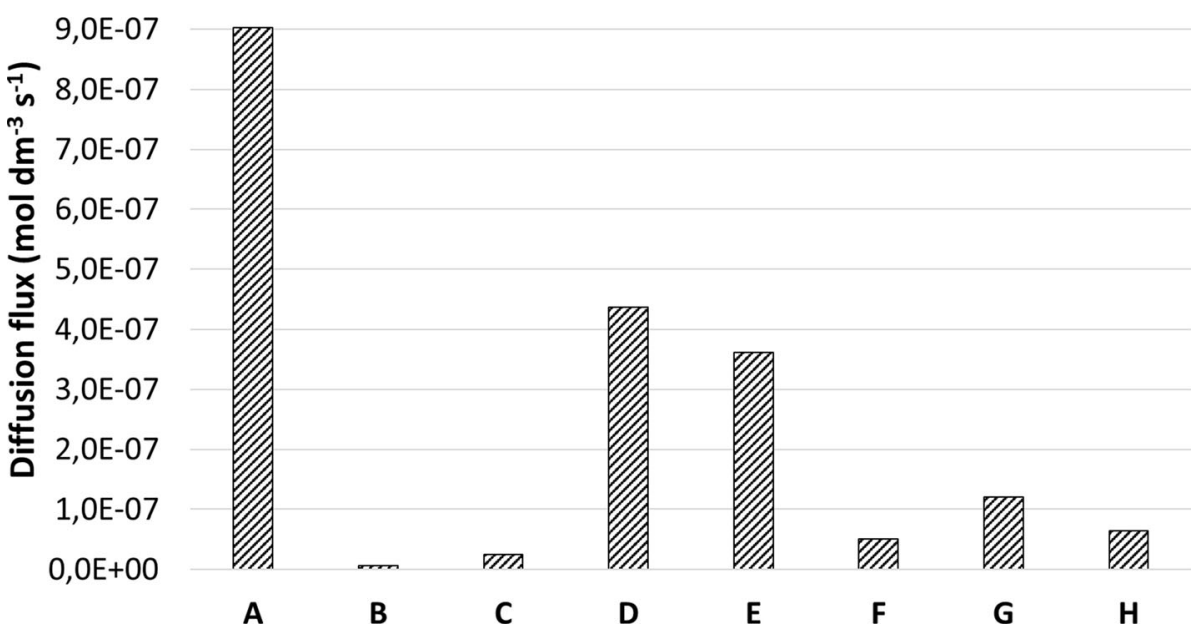




\section{Discussion}

The test showed that one layer of graphene on the PSU membrane is not enough to stop ion transport as the blocking efficiency was only $20 \%$ referring to the bare PSU membrane in a control experiment. The ions of $\mathrm{NaCl}$ could quite freely flow through the filter to pure water without changes in the liquid level in the capillaries. As it could be expected, this result confirmed the presence of defects larger than point defects in the atomic carbon network of the graphene layer. The transfer of larger graphene sheets involves a higher risk of deformations and disruptions in the graphene structure.

Unfortunately, the multiple transfer of graphene layers on PSU membrane did not bring the desired effect. Three overlapping layers of graphene were too hermetic and not permeable to water.

The use of graphene oxide was the other way of reduction in mechanical defects. It supplemented larger disconnections of layers to block ions in the process of salt fluid filtration, but point defects necessary for the passage of water molecules were saved. We managed to aggregate GO flakes on the PSU membrane in places uncovered with HSMG ${ }^{\circledR}$ (see Figs. 5, 6). Low stability of GO aggregates under the conditions of the experiment was the next problem to overcome. It is known that GO laminates are very susceptible to redispersal into water. Hence, graphene oxide flakes distributed on the HSMG ${ }^{\circledR}$ graphene layer could be flushed out by a flowing water stream during the filtration process. Moreover, the size of nanochannels in GO aggregates under the influence of water can significantly increase due to high hydrophilicity of GO nanosheets. These factors can negatively affect the overall selectivity of composite membranes.

In order to minimize the risk of degradation of the sealing during water flow, the GO layer was prepared from GO suspensions using the vacuum filtration method. This action was expected to forcefully penetrate the GO flakes deeper into the pores of the polysulfone membrane as well as better anchoring of the GO aggregates in graphene defects. Unfortunately, the pressure influence on the graphene oxide caused the GO flakes to be arranged so tightly that an impermeable layer was created.

The sealing made of GO flakes was covered with the HSMG® graphene layer once again. In the ' $\mathrm{C}$ ' specimen (GO plates between graphene sheets), high water and ions flux was gained because graphene layers not bonded between GO allow the use of porosity in GO-and defects in the graphene-to transport water as well as salt ions.

The structure of GO aggregates was strengthened by cross-linking of GO flakes with hydrazine molecules which was another proposal of GO sealing protection. Mixing GO solution with hydrazine and coating it with graphene was not effective. A better solution was to first place the GO solution, let it dry and then bond the GO with hydrazine so that the GO flakes in water/alcohol solution could settle down in graphene discontinuities on the PSU surface (using the hydrophilicity of the PSU and the hydrophobicity of graphene).

Satisfactory ion blocking as well as the GO sealing protection was achieved in the case of interfacial polymerization of nylon in defects and cracks of composite membranes. Unfortunately, nylon also effectively blocked the water flux that was confirmed by the osmosis tests for $\mathrm{F}$ and $\mathrm{H}$ membranes.

In summary, the best compromise between water permeability and the rejection rate was obtained for membrane with graphene/GO/hydrazine. Water flux of $15 \mathrm{ml} \mathrm{h}^{-1} \mathrm{~m}^{-2} \mathrm{bar}^{-1}$ is one order of magnitude lower than for commercial reverse osmosis membranes currently used [20], graphene-based membranes fabricated by $\mathrm{O}^{\prime}$ Hernet al [8] and by $\mathrm{M}$. Kafiah et al. [7]. The diffusion flux for this specimen was $5 \cdot 10^{-8} \mathrm{~mol} \mathrm{~m}^{-2} \mathrm{~s}^{-1}$, which is two orders of magnitude lower than in experiment with $\mathrm{NaCl}$ presented by $\mathrm{O}^{\prime}$ Hernet al. [8]. The diffusion flux expressed in mol dm $\mathrm{dm}^{-3} \mathrm{~s}^{-1}$ is also two orders of magnitude lower than the one presented by Kafiah et al. [7]. Most of the samples prepared in our experiment show a higher ion blocking efficiency (\%) than presented in the literature [7, 27]. Adding polyamide by interfacial polymerization on our membranes helped to decrease ion flux through the membrane, but also water could not flow. The use of hydrazine reduced the oxidative area of GO flakes and decreased the possibility of forming hydrogen bonds between water molecules and hydroxyl groups. Thus, it allowed the enhancement of water transport through the tested membranes. This result was compatible with theoretical predictions regarding the water transport mechanism, which shows that oxygen groups present in GO flakes can retain the flowing water molecules near to them and increase fluid-boundary friction [28]. 


\section{Conclusions}

New membranes containing combinations of largearea $H S M G \AA$ graphene, graphene oxide, hydrazine and polyamide on porous polysulfone scaffold were fabricated. The tenths of a nanometer size defects naturally occurring in HSMG ${ }^{\circledR}$ graphene were the basis for the design and implementation of the composite filters. GO flakes, hydrazine treatment and synthesis of interphase polymers were used to cover large discontinuities resulting mainly from the transfer process. Different sealing compositions led to different results. The composite membranes were characterized by either better ion blocking properties or revealed a greater water content under the impact of the set osmotic pressure gradient.

The optimal solution was to use a variant of sealing large HSMG ${ }^{\circledR}$ graphene defects with GO flakes treated with hydrazine. Hydrazine cross-linked and reduced the number of oxygen groups, which resulted in the improvement in the filtering properties of the entire membrane surface.

The presented composites appeared to be semipermeable membranes with excellent effectiveness in ion transport blocking. It was two orders of magnitude better than presented in the scientific literature. At the same time, both high water flux nearing one of commercial RO membranes and graphene membranes in the scientific literature were possible. The graphene-based filters proposed in this article have proven to be useful as semi-permeable membranes in water treatment.

\section{Acknowledgements}

The research was performed with the use of EU funds within the framework of the project no. POIR.04.01.04-00-0089/15, Measure 4.1 "Scientific research and development works," Sub-measure 4.1.4 "Application projects" of the Smart Growth Operational Programme, 2014-2020, project title: "Graphene based composite materials for water purification." The authors thank Magdalena Kucińska (Bionanopark Sp. zo.o.) for conducting HR TEM examination, Dorota Nowak for conducting Raman examination, Bartłomiej Januszewicz for conducting SEM examination, Radomir Atraszkiewicz and Piotr Zawadzki for performing the graphene synthesis.

\section{Compliance with ethical standards}

Conflict of interest The authors report no conflicts of interest associated with this publication.

Open Access This article is licensed under a Creative Commons Attribution 4.0 International License, which permits use, sharing, adaptation, distribution and reproduction in any medium or format, as long as you give appropriate credit to the original author(s) and the source, provide a link to the Creative Commons licence, and indicate if changes were made. The images or other third party material in this article are included in the article's Creative Commons licence, unless indicated otherwise in a credit line to the material. If material is not included in the article's Creative Commons licence and your intended use is not permitted by statutory regulation or exceeds the permitted use, you will need to obtain permission directly from the copyright holder. To view a copy of this licence, visit http://creativecommons.org/licen ses/by $/ 4.0 /$.

\section{References}

[1] FAO (2007) Coping with water scarcity: challenge of the twenty-first century, United Nations, New York

[2] Boretti A, Al-Zubaidy S, Vaclavikova M, Al-Abri M, Castelletto S, Mikhalovsky S (2018) Outlook for graphenebased desalination membranes. npj Clean Water 1:5-11

[3] Miller JE (2003) Review of water resources and desalination technologies. US Department of Energy, United States

[4] Lee A, Elam JW, Darling SB (2016) Membrane materials for water purification: de-sign, development, and application. Environ Sci Water Res Technol 2:17-42

[5] Xu G, Xu J, Su H, Liu X, Lu-Li ZH, Feng H, Das R (2019) Two-dimensional (2D) nanoporous membranes with subnanopores in reverse osmosis desalination: latest developments and future directions. Desalination 451:18-34

[6] Scott Bunch J, Verbridge S, Alden J, van der Zande A, Parpia J, Craighead H, McEuen P (2008) Impermeable atomic membranes from graphene sheets. Nano Lett 8:2458-2462

[7] Kafiah F, Khan Z, Ibrahim A, Karnik R, Atieh M, Laoui T (2016) Monolayer graphene transfer onto polypropylene and polyvinylidene difluoride microfiltration membranes for water desalination. Desalination 388:29-37

[8] O’Hern SC, Jang D, Bose S, Idrobo JC, Song Y, Laoui T, Kong J, Karnik R (2015) Nanofiltration across defect-sealed nanoporous monolayer graphene. Nano Lett 15:3254-3260 
[9] Girit CO, Meyer JC, Erni R, Rossell MD, Kisielowski C, Yang L, Park CH, Crommie MF, Cohen ML, Louie SG, Zettl A (2009) Graphene at the edge: stability and dynamics. Science 323(5922):1705-1708

[10] Lee C, Wei X, Kysar JW, Hone J (2008) Measurement of the elastic properties and intrinsic strength of monolayer graphene. Science 321(5887):385-388

[11] Stetson JB, Mercurio J, Rosenwinkel A, Bedworth PV (2012) Perforated graphene deionization or desalination, US20120048804A

[12] Celebi K, Buchheim J, Wys R, Droudian A, Gasser P, Shorubalko I, Kye J, Lee C, Park H (2014) Ultimate permeation across atomically thin porous graphene. Science 344:289-292

[13] Surwade SP, Smirnov SN, Vlassiouk IV, Unocic RR, Veith GM, Dai S, Mahurin SM (2015) Water desalination using nanoporous single-layer graphene. Nat Nanotechnol 10:459-464

[14] Koenig S, Wang L, Pellegrino J, Bunch J (2012) Selective molecular sieving through porous graphene. Nat Nanotechnol 7:728-732

[15] O’Hern S, Idrobo J, Song Y, Kong J, Laoui T, Atieh M, Boutilier M, Karnik R (2014) Selective ionic transport through tunable subnanometer pores in single-layer graphene membranes. Nano Lett 14:1234-1241

[16] Kula P, Rzepkowski A, Pietrasik R, Atraszkiewicz R, Dybowski K, Modrzyk W (2016) Method of producing graphene from liquid metal, US 9284 640, USA

[17] Dybowski K, Kowalczyk P, Kula P, Jeziorna A, Atraszkiewicz R, Kołodziejczyk $Ł$, Zawadzki P, Nowak D, Kaźmierczak T, Kucińska M (2018) Graphene-based composite membrane for water desalination. Arch Metall Mater 63:1379-1383

[18] Flowers LC, Berg D (1969) Graphitic oxide membrane for desalinating water, US3457171A

[19] Chu B, Hsiao BS, Ma H, Wang Z (2016) Porous graphene based composite membranes for nanofiltration, desalination, and pervaporation, WO2016011124A1

[20] Tsou CH, An QF, Lo SC, De Guzman M, Hung WS, Hu CC, Lee KR, Lai JY (2015) Effect of microstructure of graphene oxide fabricated through different self-assembly techniques on 1-butanol dehydration. J Membr Sci 477:93-100

[21] Kim HW, Yoon HW, Yoon SM, Yoo BM, Ahn BK, Cho YH, Shin HJ, Yang H, Paik U, Kwon S, Choi JY, Park HB (2013) Selective gas transport through few-layered graphene and graphene oxide membranes. Science 342:91-95

[22] Giménez-Pérez A, Bikkarolla SK, Benson J, Bengoa C, Stüber F, Fortuny A, Fabregat A, Font J, Papakonstantinou P (2016) Synthesis of N-doped and non-doped partially oxidised graphene membranes supported over ceramic materials. J Mater Sci 51:8346-8360

[23] Kula P, Szymański W, Kołodziejczyk Ł, Atraszkiewicz R, Dybowski K, Grabarczyk J, Pietrasik R, Niedzielski P, Kaczmarek Ł, Cłapa M (2015) High strength metallurgical graphene-mechanism of growth and properties. Arch Metall Mater 60(4):2535-2541

[24] Wang Y, Zheng Y, Xu X, Dubuisson E, Bao Q, Luoraz J, Loh KP (2011) Electrochemical delamination of CVDgrown graphene film: toward the recyclable use of copper catalyst. ACS Nano 5(12):9927-9933

[25] Dybowski K, Romaniak G, Kula P, Jeziorna A, Kowalczyk P, Atraszkiewicz R, Kołodziejczyk Ł, Nowak D, Zawadzki P, Kucińska M (2019) Impact of the method of separating graphene from the growth substrate on the quality of the $2 \mathrm{D}$ material obtained. Arch Metall Mater 64(4):131-1326

[26] Kaczmarek Ł, Kula P, Warga T, Kołodziejczyk Ł, Louda P, Borůvková K, Niedzielski P, Szymański W, Volenský L, Pawłowski W, Zawadzki P (2019) Creation of a 3D structure based on the high strength metallurgical graphene ${ }^{\circledR}$. Surf Rev Lett 26(6):1850206

[27] Pendergast MM, Hoek EMV (2011) A review of water treatment membrane nanotechnologies. Energy Environ Sci 4:1946-1971

[28] Chen B, Jiang H, Liu X, Hu X (2017) Observation and analysis of water transport through graphene oxide interlamination. Phys Chem C 121:1321-1328

Publisher's Note Springer Nature remains neutral with regard to jurisdictional claims in published maps and institutional affiliations. 